\title{
ATP-measurements in estimation of effect possessing by humic compounds on various cells of microorganisms
}

Stepanov N., Senko O., Efremenko E.

Lomonosov Moscow State University, Moscow, Russia, elena_efremenko@gmail.com

Emanuel Institute of biochemical physics RAS, Moscow, Russia

doi: 10.36291/HIT.2019.stepanov.079

The effectiveness of using the estimation of adenosine triphosphate (ATP) concentration in cells to control the cell viability of microorganisms in a wide variety of biocatalytic processes has been repeatedly demonstrated [1-4]. The ATP concentration can be quickly and easily determined using bioluminescence with great accuracy, even with an ultralow content of the substance in the sample (as low as $10^{-14} \div 10^{-12} \mathrm{M}$ ), using luciferin-luciferase reagents $[4,5]$. However, when using ATP-analysis to determine the energy status of cells in any new biocatalytic process, it is necessary to evaluate the possible effect of components of the culture media on the activity of the applied samples of luciferase, since it is known that some substances can inhibit this enzyme [5].

In a study of the use of humic compounds (HCs) for suppression of biocatalytic gas evolution from landfills, it was proposed to use the ATP-analysis to assess the viability of cells of anaerobic microorganisms involved in the process of gas formation [6]. For this, the inhibitory effect of $\mathrm{HCs}$ on the luciferase reagent was previously evaluated.

It was found that $\mathrm{HCs}$ in a concentration of more than $0.1 \mathrm{~g} / \mathrm{L}$ in the cultural media have a notable inhibitory effect on luciferase, and the concentrations of HCs more than 5 $\mathrm{g} / \mathrm{L}$ completely inhibit the luminescence of this enzyme.

Guided by these results, in order to adequately assess the concentration of intracellular ATP in cells exposed to presence of HCs, the analyzed samples of cultural media with $\mathrm{HCs}$ were diluted an appropriate number of times to avoid inhibition of luciferase within analysis. A positive correlation between the metabolic activity of cells in biogas formation and the concentration of ATP in cells was observed. So, the control of ATP concentration appeared to be an attractive and simple tool for finding compounds that can suppress methanogenesis in landfills.

Acknowledgements. This research was funded by the Russian Foundation for Basic Research (Grant No. 18-29-25065).

References

1. Efremenko E.N., Tatarinova N.Y. // Microbiology. 2007. 76(3):336-341.

2. Stepanov N., Efremenko E. // New Biotechnology. 2017. 34:54-58.

3. Efremenko E.N. et al. // Int. Biodeterioration Biodegradation. 2005. 56:94-100.

4. Ismayilov I.T. et al. // Moscow University Chemistry Bulletin. 2015. 70(4):197-201.

5. Lomakina G.Y. et al. // Biochemistry. 2015. 80:701-713.

6. Stepanov N. et al. // Sustainability. 2019. 11:3158-11p. 